J. Clin. Chem. Clin. Biochem.

Vol. 14, 1976, pp. 557-559

\title{
Erythropoetin-Exkretion im alkalischen und sauren Harn beim Menschen ${ }^{1}$ )
}

\author{
Von E. Schulz und H. Cissée \\ Medizinische Universitäts-Poliklinik und Medizinische Klinik(Direktor: Prof. Dr. W. Kaufmann) Köln-Merheim
}

(Eingegangen am 24. April/25. August 1976)

Zusammenfassung: Unter einer Harnalkalisierung mit $\mathrm{NaHCO}_{3}$ stieg bei 2 anämischen Patienten von Marver \& Gurney ((1968), Ann. N. Y. Acad. Sci. 149, 570-575) die Erythropoetin-Exkretion um das 17-bzw. 3,5-fache des Ausgangswertes an. Wir erzielten bei 6 Versuchspersonen unter einer täglichen Gabe von $3,5 \mathrm{~g} \mathrm{NH}_{4} \mathrm{Cl}$ durchschnittliche Harn-pHWerte von 5,9 und unter einer harnalkalisierenden Medikation von Hexakaliumhexanatriumpentacitrat einen pH-Wert von 7,5. Im alkalischen Harn fanden wir jedoch durchschnittlich nur eine nicht signifikante Zunahme der Erythropoetin-Exkretion um das Doppelte. Nur 2 Versuchspersonen zeigten einen signifikanten Anstieg.

\section{Excretion of erythropoietin by humans during the production of alkaline or acidic urine}

Summary: Marver \& Gurney ((1968) Ann. N. Y. Acad. Sci. 149, 570-575) reported that the erythropoietin excretion in two anaemic patients increased 17 and 3.5-fold, respectively, during the production of alkaline urine by the administration of $\mathrm{NaHCO}_{3}$. We have studied the excretion of erythropoietin in 6 persons, following the daily administration of $3.5 \mathrm{~g}$ of $\mathrm{NH}_{4} \mathrm{Cl}$, which resulted in an average urine $\mathrm{pH}$ of 5.9; and following the administration of hexapotassium hexasodium pentacitrate, which increased the urine $\mathrm{pH}$ to 7.5 . In the alkaline urine, erythropoietin excretion showed an average, but non-significant, two-fold increase. The increase was significant in only two experimental persons.

\section{Einfihrung}

Die große Variabilität der Erythropoetin-Exkretion im Harn ist weitgehend ungeklärt. Shore et al. fanden in acht aufeinanderfolgenden 6-stündigen Sammelperioden bei einem anämischen Patienten eine Streuung um das 30 fache (von 77 bis $2150 \mathrm{E} / 6$ Stunden). In einer Gruppe von Normalpersonen schwankte die Erythropoetin-Exkretion von 24stündigen Sammelperioden nach Finne um das 14 fache.

Marver \& Gurney sahen bei einem nierengesunden Anämiker nach Verabfolgung von $6 \mathrm{~g} / \mathrm{d}$ Natriumhydrogencarbonat einen Anstieg des Harn-pH von 5 auf 8 und eine Zunahme der Erythropoetin-Exkretion um das 17 fache des Ausgangswertes. Ein żweitèr anämischer Patient zeigte bei einem Anstieg des pH-Wertes von 6,3 auf 7,4 einen Anstieg der Erythropoetin-Exkretion um das 3,5fache. Wir überprüften diese Befunde an $6 \mathrm{Ver}$ suchspersonen, um die Bedeutung des Harn-pH's auf die Erythropoetin-Exkretion zu erfassen.

\section{Methodik}

Patienten und Untersuchungsmaterial

Zur Stimulierung der Erythropoetin-Exkretion gaben wir einem Nierenpatienten (Serumkreatinin $26 \mathrm{mg} / \mathrm{l}=230 \mu \mathrm{mol} / \mathrm{l}, \mathrm{C}_{\mathrm{Kreat}}$

\footnotetext{
1) Mit Unterstützung der Deutschen Forschungsgemeinschaft.
}

$45 \mathrm{ml} / \mathrm{min}$, Hämoglobin $140 \mathrm{~g} / \mathrm{l}=8,7 \mathrm{mmol} / \mathrm{l}$ ) und vier gesunden Männern im Alter zwischen 23 und 34 Jahren über drei Wochen das Androgen Fluoxymesteron täglich in einer Dosis von $40 \mathrm{mg} / \mathrm{m}^{2}$ Körperoberfläche und alkalisierten am Ende der dritten Woche den Harn mit einer Tagesdosis von $25 \mathrm{~g}$ Hexakaliumhexanatriumpentacitrat (Uralyt U, Firma Dr. Madaus, Köln). Dieser alkalische Harn wurde bei $+4^{\circ} \mathrm{C}$ gesammelt und tiefgefroren. Nach Normalisierung des Harn-pH's gaben wir anschließend den vier gesunden Männern $3 \mathrm{~g} \mathrm{NH}_{4} \mathrm{Cl}$ über einen Tag verteilt zum Ansäuern des Harns. Unter $\mathrm{NH}_{4} \mathrm{Cl}$ wurde ebenfalls ein 24-Stunden-Harn gesammelt und eingefroren. Bei dem Nierenpatienten W. E. verzichteten wir wegen des spontan niedrigen Harn-pH's auf das Ansäuern. Eine 68jährige Patientin mit aplastischer Anämie, einem Hämoglobin von $44 \mathrm{~g} / \mathrm{l}=2,67 \mathrm{mmol} / \mathrm{l}$ und einem Hämatokrit von 0,40 untersuchten wir in gleicher Weise ohne Androgenstimulierung. Nach dem Auftauen bestimmten wir den Harn-pH und engten den Urin auf $60 \mathrm{ml}$ durch Harndialyse (4) ein.

Erythropoetinbestimmung

Die Erythropoetinbestimmung erfolgte mit der posthypoxischen polyglobulen weiblichen NMRI-Maus mit Hilfe der ${ }^{59}$ Eisenincorporationsrate $\left({ }^{59} \mathrm{Fe}-\mathrm{IRC}\right)$, die nach folgender Formel berechnet wurde:

$\%^{59} \mathrm{Fe}-\mathrm{ICR}=\frac{\text { Radioaktivität der Probe } \cdot \text { Tiergewicht }[\mathrm{g}] \cdot 8,7}{\text { Injizierte Radioaktivität } \cdot \text { Volumen der Probe }[\mathrm{ml}]}$

Der Faktor 8,7 resultiert aus dem Blutvolumen von $8,7 \mathrm{ml} /$ $100 \mathrm{~g}$ Maus (5).

Die bei Lieferung 8 Tage alten NMRI-Mäuse (Firma Ivanovas, Kißlegg/Allgäu) bekamen 5 Tage vor Eintritt in die Unterdruckkammer $5 \mathrm{mg}$ Eisen als Eisendextran (Myofer, Hoechst) i. m. Sie verweilten drei Wochen lang 16 Stunden täglich in der Unter- 
druckkammer bei $0,4 \mathrm{Atm}(=310 \mathrm{~mm} \mathrm{Hg} .=7010 \mathrm{~m}$ simulierte Höhe). Das Injektionsschema erfolgte in Anlehnung an Alexanian (6), wobei wir jeder Maus $3 \times 2$ ml Harnkonzentrat bzw. $3 \times 1$ $\mathrm{ml}$ Plasma intraperitoneal injizierten. Gemessen wurde die 66Stunden- ${ }^{59} \mathrm{Fe}-\mathrm{ICR}$ (Abb. 1).

Zur Bestimmung jeweils eines Erythropoetin-Wertes verwandten wir eine Gruppe von acht randomisierten Mäusen. Eine Charge von 64 Mäusen wurde aufgeteilt in 6 Gruppen zur Ermittlung der Patientenwerte, eine Kontrollgruppe, die $3 \times 1 \mathrm{ml}$ physiologische $\mathrm{NaCl}$-Lösung und eine weitere Gruppe, die ErythropoetinStandard der Firma Connaugh bzw. den internationalen Standard B der WHO intraperitoneal bekamen.

Am ersten posthypoxischen Tag betrug der Hämatokrit $0,75 \pm 0,04$ und die ${ }^{59} \mathrm{Fe}-\mathrm{ICR} 71 \pm 5$, am 10 . posthypoxischen der Hämatokrit $0,66 \pm 0,04$ und die ${ }^{59} \mathrm{Fe}-\mathrm{ICR}$ bei den Kontrollen unter physiologischer $\mathrm{NaCl}-\mathrm{L}$ ösung $0,27 \pm 0,1 \%(\overline{\mathrm{x}} \pm \mathrm{s})$.

\section{Standardkun'e}

Insgesamt 119 posthypoxische polyglobule Mäuse erhielten den Standard B in einer Verdünnung von 0,05 bis $6 \mathrm{E}$./Maus zur Erstellung einer Standard-Dosiswirkungskurve, so daß die gemessenen Eisenincorporationsraten in Erythropoetineinheiten ausgedrückt werden können (Abb. 2). Bei der Berechnung der Regressionskurve wurde die Methode der kleinsten Quadrate angewandt und das Ausgleichspolynom 5. Grades berechnet. Die Regressionsrechnungen verdanken wir Herrn Dipl.-Mathematiker J. Vollmar, Mannheim.

\section{Ergebnisse}

Die Abbildung 3 gibt die Harn-pH-Werte der Sammelurine und die 24-Stunden-Erythropoetin-Exkretion wieder.

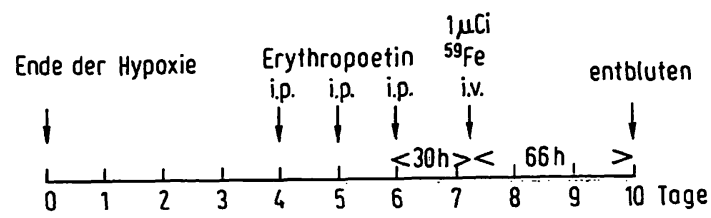

Abb. 1. Injektionsschema bei der posthypoxischen polyglobulen Maus zur Erythropoetin-Bestimmung.

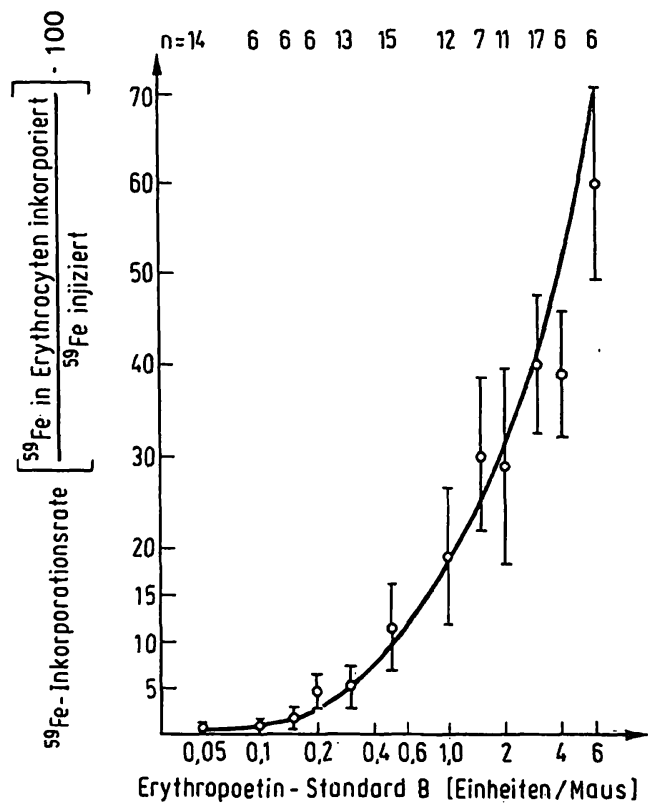

Abb. 2. Dosiswirkungskurve für Erythropoetin-Standard B der WHO, erstellt an insgesamt 119 polyglobulen Mäusen $(\overline{\mathbf{x}} \pm s)$.

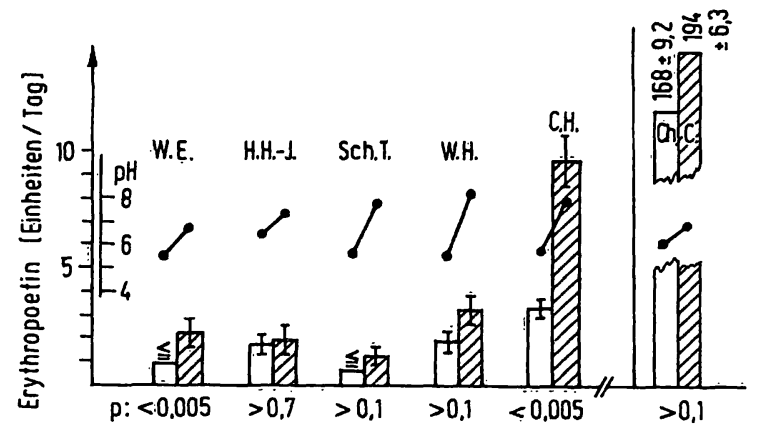

Abb. 3. Ërythropoetin-Exkretion uninter Veränderung des HarnpH-Wertes bei einem Nierenpatienten (W. E.), vier gesunden Männern und einer Patientin mit aplastischer Anämie (Ch. C.) $\left(\overline{\mathrm{X}} \pm s_{\overline{\mathrm{X}}}\right.$ aus einer Bestimmung mit Hilfe von 8 Mäusen). Die Erythropoetin-Einheiten sind auf den internationalen Standard B der WHO bezogen (vgl. Abb. 2). - Harn-pH, offene Säulen nach $\mathrm{NH}_{4} \mathrm{Cl}$ $(3 \mathrm{~g} / \mathrm{d})$, schraffierte Säulen nach Uralyt U ( $25 \mathrm{~g} / \mathrm{d})$.

Die Harn-pH-Werte liegen unter der Alkalimedikation durchschnittlich bei 7,5 und unter $\mathrm{NH}_{4} \mathrm{Cl}$ bei 5,9. Im alkalischen Ham findet sich bei allen Versuchspersonen eine höhere Erythropoetin-Ausscheidung als im sauren Milieu. Der Anstieg ist jedoch nur bei W. E. und C. H. signifikant. Die Zunahme der Erythropoetin-Exkretion bei alkalischem Harn beträgt bei den Versuchspersonen W. E. $138 \%$, Sch. T. $100 \%$, H. H.-J. $8 \%$, W. H. $79 \%$, C. H. $191 \%$ und bei Ch. C. $15 \%$.

Alle nichtanämischen Versuchspersonen zusammen zeigen nach Alkalisierung des Harns mit einer durchschnittlichēn Erythropoetin-Ausscheidung von 3,6 E./24 Stunden eine um das Zweifache höher liegende ErÿthropoetinExkretion im Vergleich zur mittleren ErythropoetinAusscheidung im sauren Harn (1,7 E./24 Stunden). Der Anstieg ist jedoch nicht signifikant $(p>0,2)$. Die anämische Patientin mit einer hohen ErynthropoetiExkretion und einer Zunahme von 168 auf 194 E./24 Stunden weist ebenfalls keinen Unterschied auf.

Um die spontanen pH-Wertschwankungen im Harn zu erfassen, wurde über einen Zeitraum von 5 Wochen bei allen Personen einmal wöchentlich der Harn-pH in einem 24-Stunden-Harn ohne Einfluß von Medikamenten bestimmt. Der durchschnittliche pH-Wert lag bei dem Nierenpatienten W. E. bei $5, \overline{7} \pm 0,1$ (VK $=2,5 \%$ ), und bei den fün übrigen Versuchspersonen bei $6,2 \pm 0,3$ $(\mathrm{VK}=5,4 \%)$. Die Differenz zwischen dem höchsten und dem niedrigsten pH-Wert betrug bei Nierenpatienten 0,3 und bei den übrigen 5 Versuchspersonen 0,8 bis 0,9 . Bei einer Versuchsperson wurde der Harn-pH-Wert über einen Zeitraum von einem Jahr 16mal kontrolliert, wobei der Variationskoeffizient mit 1,6\% ebenfalls sehr niedrig lag.

Bei den fünf nịcht anämischen Versuchspersonen bestimmten wir auch die Erythropoetin-Konzentration im Plasma. Nach Injektion von Plasma in die Mäusé 
lag die durchschnittliche ${ }^{59} \mathrm{Fe}-\mathrm{ICR}$ bei $0,7 \pm 0,2 \%$ $(\bar{x} \pm s)$. Die ${ }^{59} \mathrm{Fe}$-ICR bei den entsprechenden Kontrollen unter physiologischer $\mathrm{NaCl}$-Lösung betrug $0,3 \pm 0,1 \%$ $(\bar{x} \pm s)$. Der Unterschied war signifikant, $(p<0,005)$. Mit Hilfe der Standard-Dosiswirkungskurve (Abb. 2) ergaben sich für diese Versuchspersonen folgende Erythropoetin-Konzentrationen im Plasma: W. E. 20, Sch. T. 30, H. H.-J. 23, W. H. 38 und C. H. 28 E/l. Die ErythropoetinKonzentration im Plasma zeigte keine auffallige Korrelation zur Erythropoetin-Exkretion der dazugehörigen Versuchsperson.

\section{Besprechung der Ergebnisse}

Nach unseren Befunden kommt der Harn-pH-Veränderung keineswegs die Bedeutung $\mathrm{zu}$, wie es nach den Untersuchungen von Marver \& Gurney (3) den Anschein hat. Bei einem vergleichbaren $\mathrm{pH}$-Anstieg von durchschnittlich 5,9 auf durchschnittlich 7,5 lag die ErythropoetinExkretion zwar bei allen sechs Versuchspersonen im alkalischen Harn höher als im sauren, der Unterschied war jedoch nur bei zwei Personen signifikant. Im Vergleich dazu zeigte der erste Patient von Marver \& Gumey (3) eine Zunahme der Erythropoetin-Ausscheidung um das 17 fache des Ausgangswertes und der zweite um das 3,5fache. Unsere Befunde bestätigen die Mitteilung von Marver \& Gurney (3) nur insofern, als die SäureBasen-Balance in einzelnen Fällen einen Einfluß auf die Erythropoetin-Ausscheidung ausüben kann. Wir finden jedoch bisher keine Erklärung für den bei vier von unseren sechs Versuchspersonen fehlenden bzw. geringen Effekt der Harnalkalisierung auf die Erythropoetin-Exkretion im Vergleich zu dem starken Effekt bei beiden Patienten von Marver \& Gurney (3), die untereinander allerdings auch erheblich differieren. Die beiden von
Marver \& Gurney (3) untersuchten Patienten waren stark anämisch mit einer Hämoglobin-Konzen tration von 75 bzw. $45 \mathrm{~g} / 1$. Bemerkenswert erscheint, daß der Anstieg der Erythropoetin-Exkretion unserer Patientin Ch. C. mit einer vergleichbar starken Anämie nicht signifikant ist. Ob die Art des von uns zur Harnalkalisierung gewählten Mittels (Hexakaliumhexanatriumpentacitrat) gegenüber dem von Marver \& Gurney (3) eingesetzten Natriumhydrogencarbonat einen Einfluß auf die Höhe der Erythropoetin-Exkretion ausübt, läßt sich nur durch vergleichende Untersuchungen klären. Die Erythropoetin-Stimulation mit Fluoxymesteron erfolgte bei unseren Versuchspersonen lediglich zum besseren Nachweis des Erythropoetins im Harn. Unter der angewandten Fluoxymesterondosis steigt die Erythropoetin-Exkretion bei Männern nach Alexanian auf das 8 fache des Ausgangswertes an (7).

$\mathrm{Zu}$ den von Marver \& Gurney (3) unternommenen interessanten, aber rein hypothetischen Erklärungsversuchen der verstärkten Erythropoetin-Exkretion im alkalischen Harn über eine ,nonionic diffusion" soll nichts hinzugefügt werden, solange die biochemischen Kenntnisse über Erythropoetin so unvollständig sind. Eine Instabilität von Erythropoetin bei saurem pH-Wert des Harns wurde von Marver \& Gurney (3) überprüft und ausgeschlossen.

Die spontanen $\mathrm{pH}$-Wertschwankungen im 24-StundenHarn sind bei einer mehrwöchigen Beobachtungsperiode entsprechend den oben angegebenen Variationskoeffizienten von 1,6 bzw. 2,5\% bei Einzelpersonen oder $5,4 \%$ im Kollektiv sehr gering. Auch aus diesem Grunde sind die Harn-pH-Veränderungen kein entscheidender Faktor für die von Shore et al (1) sowie von Finne (2) nachgewiesene Variabilität in der Erythropoetin-Exkretion.

\section{Literatur}

1. Shore, N. A., Ortega, J. A. \& Hammond, G. D. (1970), Variability of erythropoietin excretion in the urine of patients with anemia. XIII. Internat. Kongr. Hematol. 2.-8. 8. 1970, München, Abstr. p. 11.

2. Finne, P. H. (1968), Ann. N. Y. Acad. Sci. 149, 497-503.

3. Marver, D. \& Gurney, C. W. (1968), Ann. N. Y., Acad. Sci. $149,570-575$.

4. Schulz, E. \& Cissee, H. (1976), this j, 14, 537-542.
5. Schulz, E. (1972), Erythropoetin (ESF) im Serum und Harn bei chronischer Niereninsuffizienz im Stadium der kompensierten Retention unter hypoxischer Știmulation und Androgengabe. Habilitationsschrift Köln.

6. Alexanian, R. (1966), Blood 28, 344-353.

7. Alexanian, R., Vaughn, W. K. \& Ruchelman, M. W. (1967), J. Lab. Clin. Med. 20, 777-785.

Privatdozent Dr. Ekkehard Schulz Vinzenz-Pallotti-Hospital Innere Abteilung Vinzenz-Pallotti-Str. 20-24

D-5060 Bergisch-Gladbach/Bensberg 


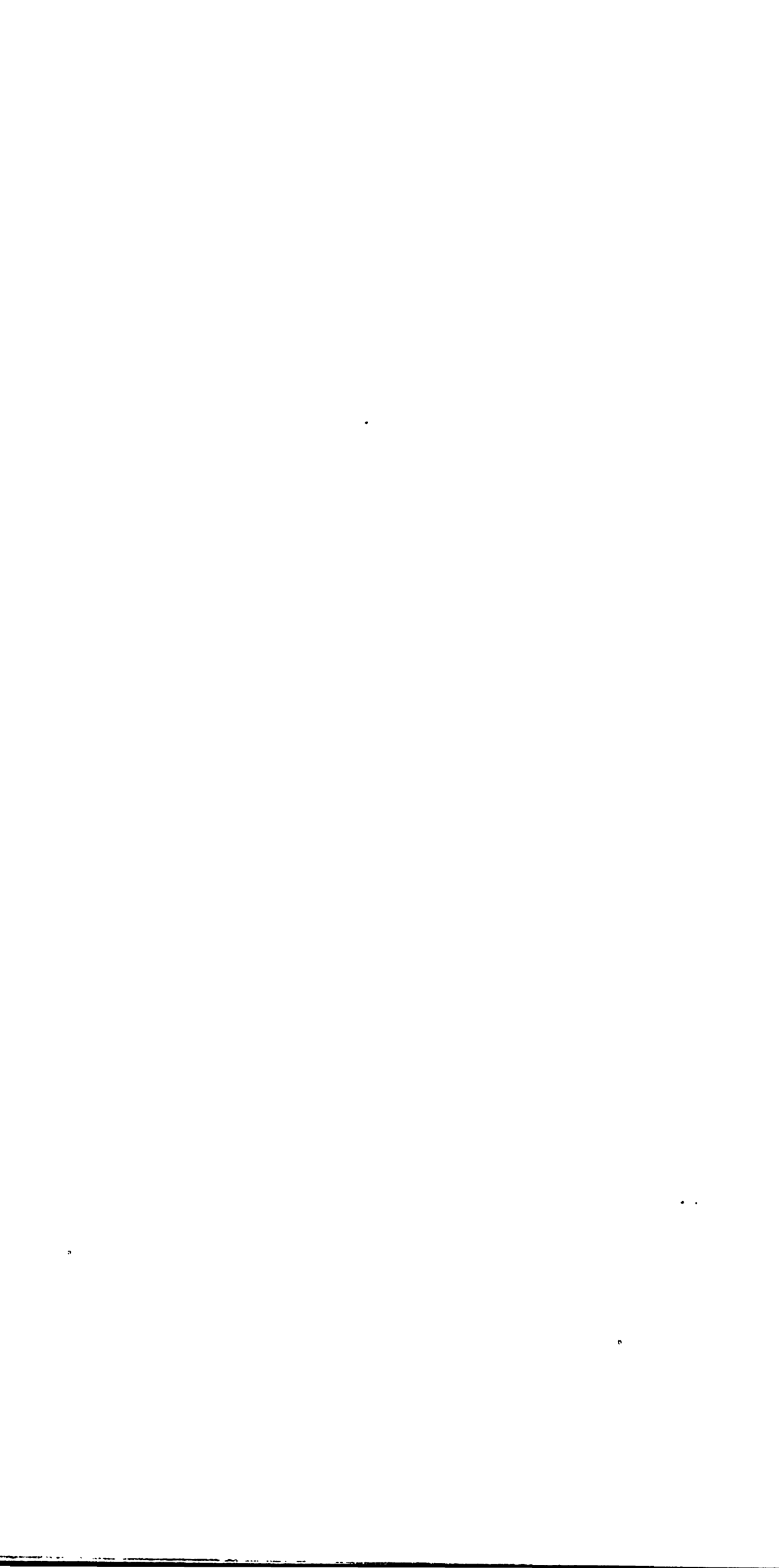

